\title{
Évaluation d'indicateurs fécaux pour prédire la digestibilité et les quantités ingérées de Dichanthium sp par des bovins créoles
}

\author{
M Boval ${ }^{1}$, JL Peyraud ${ }^{2 *}$, A Xande ${ }^{1}$, G Aumont ${ }^{1}$, \\ O Coppry ${ }^{1}$, G Saminadin ${ }^{1}$ \\ ${ }^{1}$ Station de zootechnie, Inra, BP 1232, 97185 Pointe-à-Pitre cedex; \\ 2 Station de recherches sur la vache laitière, Inra, 35590 Saint-Gilles, France
}

(Reçu le 21 juin 1994 ; accepté le 21 août 1995)

\begin{abstract}
Résumé - Un essai de digestibilité a été réalisé en continu pendant 53 jours avec six taurillons créoles à l'auge, afin d'établir pour Dichanthium sp des équations de prédiction de la digestibilité de la matière organique $(\mathrm{dMO})$ et des quantités de matière organique ingérées (MOI), à partir de la composition chimique des fèces. Les constituants qui ont permis de prédire la dMO avec la plus grande précision sont les matières azotées totales (MAT $f \% \mathrm{MO}$ ) que ce soit avec une équation de type linéaire, quadratique ou hyperbolique ( $\mathrm{dMO}=0,983-4,002 / \mathrm{MAT} f$, $\mathrm{ETR}=0,023)$. Les constituants pariétaux des fèces ont été des covariables moins précises que MATf. La quantité de MAT (en g) excrétée dans les fèces a été l'indicateur le plus précis pour prédire MOI $(E T R=240 \mathrm{~g}$ de $\mathrm{MO})$. Les équations établies ont ensuite été utilisées pour estimer la digestibilité et les quantités ingérées par des génisses créoles au pâturage sur des prairies à Dichanthium sp au cours de trois essais caractérisés par des croissances journalières des animaux très différentes $(740,430$ et $140 \mathrm{~g})$. C'est l'équation hyperbolique qui a conduit à des estimations de la digestibilité et des quantités de MOI les plus cohérentes avec la croissance et les besoins en énergie calculés des animaux dans les trois situations alors que les modèles linéaires et quadratiques ont conduit à des biais non négligeables.
\end{abstract}

indicateurs fécaux / digestibilité / quantités ingérées / Dichanthium sp

Summary - Evaluation of faecal indicators to predict digestibility and voluntary intake of Dichanthium sp by cattle. In vivo digestibility trials involving creole bull calves were carried out in order to evaluate the potential of faecal indicators for assessing organic matter digestibility (dMO) and organic matter intake (MOI). The evaluated faecal indicators were crude protein (MAT), neutral detergent fiber (NDFI), acid detergent fiber (ADFf), residual nitrogen in ADF (NADFI) and in vitro dry matter digestibility (DIV). A continuous digestibility trial lasting 53 days was conducted with Dichanthium sp harvested from 20 to 73 days of regrowth. Six creole bull calves (256 $\pm 32 \mathrm{~kg}$ ) housed in metabolism

* Correspondance et tirés à part 
cages were fed ad libitum twice daily. Refusals and faeces were collected and weighed every day for each animal. Dry matter (MS), MAT, NDF, ADF, NADF content and DIV were determined by standard procedures from forage and faeces dried samples. Mean values were calculated per week (5 days) period for each animal. MOI was $4.88 \mathrm{~kg} /$ day and dMO ranged from 0.68 to 0.56 . In our conditions, the best predictor of dMO was MATT content. Linear, quadratic and hyperbolic functions were of similar accuracy ( $r$ sd =0.025). NDFf, ADFf and NADFf were less accurate. Faecal MAT output ( $g /$ day) was the best predictor of $\mathrm{MOI}(\mathrm{rsd}=240 \mathrm{~g}$ ). The best accurate regressions predicting dMO or $\mathrm{MOI}$ were used to estimate digestibility and intake of tethered creole heifers grazing Dichanthium sp pastures during three trials where daily body live-weight gain were different $(740,430$ and $140 \mathrm{~g})$. Only the hyperbolic model predicting $d M O(d M O=0.983-4.002 / M A T, E T R=0.023)$ allowed estimates of $M O l$ and energy intake consistent with the average daily gain and energy requirements of the grazing heifers in all trials. This model should be preferred.

\section{faecal indicators / digestibility / intake / Dichanthium sp}

\section{INTRODUCTION}

La quantité de fourrage ingérée est le principal facteur qui régit la production des ruminants au pâturage. La méthode la plus utilisée pour estimer les quantités ingérées au pâturage nécessite l'estimation de la quantité de fèces émise et de la digestibilité du fourrage ingéré (Streeter, 1969 ; Greenhalgh, 1982). L'excrétion fécale est estimée au pâturage soit par la récolte totale des fèces émises (Cordova et al, 1978) où, plus souvent, par l'utilisation d'un marqueur indigestible (Le Du et Penning, 1982).

Plusieurs méthodes indirectes permettent d'apprécier la digestibilité du fourrage ingéré au pâturage (Streeter, 1969 ; Scales et al, 1974 ; Cordova et al, 1978 ; Holechek et al, 1982 ; Judkins et al, 1989 ; Doves et Mayes, 1991). Parmi elles, celle des «indicateurs fécaux" consiste à établir avec des animaux maintenus à l'auge des équations de régression entre la composition chimique des fèces et la digestibilité du fourrage ingéré. Lancaster (1949a, cité par Streeter, 1969) a été le premier à prédire la digestibilité au pâturage à partir de la concentration en azote des fèces. Depuis, de nombreuses équations de prédiction de la digestibilité à partir de l'azote fécal ont été publiées aussi bien pour des fourrages tempérés que tropicaux (Streeter, 1969 ; Cordova et al, 1978 ; Holechek et al, 1982 ; Barthiau-Thill et Oger, 1986). Les constituants pariétaux des fèces ont aussi été utilisés comme des indicateurs de la digestibilité du fourrage ingéré (Raymond et al, 1954 ; Jarrige, 1965 ; Vera, 1973 ; Holloway et al, 1981; Guerin et al, 1988). Certains auteurs ont aussi publié des équations de prédiction directe des quantités ingérées en fonction des quantités de fèces excrétées ou de la quantité totale d'azote excrétée (Hutton et Jury, 1964 ; Cordova et al, 1978 ; Holloway et al, 1981 ; Leite et Stuth, 1990).

Les équations établies par les différents auteurs sont cependant très variables et celles établies par espèce végétale et dans les conditions dans lesquelles elles doivent s'appliquer sont les plus fiables (Greenhalgh et Corbett, 1960b ; Milford et Minson, 1965 ; Chenost, 1985). L'objectif de cet essai a été de rechercher pour Dichanthium sp, graminée prédominante dans les savanes sèches guadeloupéennes, les constituants fécaux qui permettent d'établir, d'une part, des équations de prédiction de la digestibilité de la matière organique (dMO) et, d'autre part, des équations de prédiction directe des quantités de matière organique ingérées (MOI) par des taurillons créoles, afin de pouvoir les utiliser ultérieurement au pâturage. 
MATÉRIEL ET MÉTHODES

\section{Principe du dispositif expérimental}

Six taurillons créoles ont été alimentés avec du Dichanthium sp proposé vert pendant 53 jours consécutifs. Le fourrage distribué aux taurillons a évolué en continu passant de 20 à 73 jours d'âge de repousse. Les quantités ingérées et excrétées ont été mesurées pendant 5 jours consécutifs par semaine, durant 8 semaines. Chaque période de mesure de 5 jours a été séparée de la suivante par 2 jours.

\section{Conduite des parcelles et des animaux}

Une parcelle mécanisable de Dichanthium sp de 1,5 ha a été fauchée et fertilisée ( $90 \mathrm{~kg} \mathrm{~N} / \mathrm{ha}$ en un apport) 3 semaines avant le début des mesures. Une irrigation a été effectuée en fonction de la pluviométrie et à $100 \%$ de l'évapo-transpiration maximale, pour éviter tout déficit hydrique.

Six taurillons créoles âgés de 18 mois et pesant $256 \mathrm{~kg}$ en moyenne ont été placés dans des cages individuelles, à l'abri du soleil. Les animaux ont disposé d'eau à volonté dans des abreuvoirs automatiques et de sels minéraux (manganèse : $0,75 \%$; cuivre : $0,15 \%$; zinc : $0,9 \%$; chlorure de sodium : $98,2 \%$ ) sous forme de pierre à lécher. Les animaux ont été vermifugés (avec de l'ivermectine) au début de l'essai, traités contre les tiques (au deltaméthrine) tous les 15 jours. Ils ont été pesés chaque semaine.

\section{Mesure des quantités proposées, ingérées et excrétées}

Les mesures ont débuté après 3 semaines d'adaptation des animaux aux cages. Le fourrage a été fauché et récolté quotidiennement à partir de 6 h 30 . Il a ensuite été haché, brassé et distribué aux taurillons en deux repas, à $8 \mathrm{~h} 00$ et $13 \mathrm{~h} 00$. Les quantités proposées ont été calculées pour permettre un refus de $20 \%$. Le fourrage refusé et les fèces ont été récoltés en totalité et pesés individuellement à 7 h 30 . Des échantillons de $300 \mathrm{~g}$ représentatifs du fourrage proposé, refusé et des fèces ont été prélevés quotidiennement afin de constituer des échan- tillons moyens par taurillon et par période de mesure.

\section{Analyses chimiques}

Tous les échantillons de fourrage et de fèces ont été séchés à $80^{\circ} \mathrm{C}$ pendant 48 heures et broyés à travers une grille à mailles de $0,75 \mathrm{~mm}$. Ils ont été analysés pour déterminer leur teneur en matière organique $(\mathrm{MO})$, matières azotées totales (MAT), parois totales (NDF) et lignocellulose (ADF). L'azote contenu dans l'ADF (NADF) a été dosé pour les échantillons de fèces uniquement. La digestibilité in vitro de la matière sèche (DIV) des échantillons de fourrage a aussi été déterminée.

La teneur en cendres a été déterminée par calcination des échantillons à $550^{\circ} \mathrm{C}$ pendant 8 heures. Les teneurs en MAT et NADF ont été déterminées par la méthode de Kjeldahl. Après minéralisation de l'azote, la distillation de l'ammoniaque a été réalisée à l'aide d'un générateur de vapeur (Vapodest 4) couplé à un titrimètre automatique. Les teneurs en NDF et ADF ont été déterminées selon la méthode de van Soest (1963) automatisée à l'aide du Fiberteck M6 (Técator). La DIV a été déterminée par la méthode de Tilley et Terry (1963).

\section{Analyses statistiques}

Un taurillon a dû être éliminé pour des raisons de comportement dès la seconde semaine de mesure, et n'a pas été remplacé. Les analyses statistiques ont été réalisées avec les données collectées pour cinq taurillons au cours des huit périodes de mesure (soit 40 points). La distribution des variables, leur variance liée à l'âge de repousse du fourrage ou aux taurillons, les corrélations entre variables et les équations de prédiction de la digestibilité de la MO (dMO) et de la quantité de MO ingérée (MOI) ont été analysées avec le logiciel Sas (1988).

Pour la prédiction de la dMO, les covariables considérées ont été d'une part les teneurs en MAT, NDF, ADF et NADF des fèces (MATf, NDF $f$, ADF $f$ et NADF $f$ et d'autre part les teneurs en MAT, NDF, ADF et la DIV du fourrage proposé (MATp, NDF $p$, ADF $p$, DIV $p$ ). Pour la prédiction de $\mathrm{MOI}$, la covariable prise en compte a été la quantité de MAT des fèces (qMATf). 
Pour chacun des modèles calculés, les effets taurillons et les interactions entre les taurillons et les covariables ont été testés. Deux observations pour lesquelles les résidus étaient significativement différents de 0 ont été éliminées. Les modèles ont également été calculés en considérant les digestibilités et les compositions chimiques moyennes des fèces des cinq taurillons à chaque période (soit huit points).

\section{Validation des équations de prédiction au pâturage}

Les équations de prédiction les plus précises établies à l'auge ont été utilisées au cours de trois essais au pâturage avec des fourrages de composition et de digestibilité plus variables qu'à l'auge, afin de tester la cohérence des estimations obtenues. Pour cela les quantités ingérées d'énergie nette estimées par ces équations ont été comparées aux besoins moyens en énergie des animaux pour réaliser les croissances pondérales mesurées, très différentes d'un essai à l'autre.

Ces essais visaient à tester l'effet de différents modes de conduite au pâturage sur l'ingestion. Au cours de chaque essai, trois lots de deux génisses créoles ont été conduits au pâturage à l'attache sur une savane à Dichanthium sp (à 14 jours d'âge de repousse) selon un dispositif en carré latin $3 \times 3$. Les quantités journalières de MO émises dans les fèces ont été estimées par récolte totale et individuelle au sol, pendant 4 jours consécutifs à chaque période de mesure. Ces échantillons moyens par génisse ont été séchés $\left(48 \mathrm{~h}\right.$ à $80^{\circ} \mathrm{C}$ ), broyés (grille de $0,75 \mathrm{~mm}$ ) et analysés pour déterminer les teneurs en cendres, MAT, NDF, ADF et NADF. La digestibilité et les quantités ingérées ont été estimées en utilisant les équations de prédiction ayant les ETR les plus faibles.

Les apports en énergie nette ont été calculés en multipliant les quantités ingérées de MO par la valeur énergétique de Dichanthium $\mathrm{sp}$. Celle-ci a été estimée à partir d'une relation établie pour quatre fourrages tropicaux (Peyraud, non publié) (Dichanthium sp, Digitaria decumbens, Digitaria swazilendensis, Panicum maximum) à différents âges de repousse à partir de leur valeur alimentaire données par Aumont et al (1991) :

$$
\begin{aligned}
& \text { UFL }(/ \mathrm{kg} \mathrm{MOI})=1,55 \times \mathrm{dMO}-0,255 \\
& \left(R^{2}=0,98 ; \mathrm{ETR}=0,009\right) .
\end{aligned}
$$

Les besoins en énergie nette des génisses (UFL/kg $\mathrm{P}^{0,75}$ ) au pâturage ont été estimés selon l'équation de Troccon (1987) à partir du gain moyen quotidien (GMQ en $\mathrm{kg}$ ) réalisé au cours de chacun des essais. Ils ont été majorés de $20 \%$ pour les dépenses liées à la thermorégulation et à l'activité de pâturage (Berbigier, 1988) :

$$
\text { UFL }\left(/ \mathrm{kg} \mathrm{P}^{0,75}\right)=\left(0,044+0,0415 \times \mathrm{GMQ}^{1,4}\right) \times 1,2 .
$$

Les GMQ ont été de 743,436 et $139 \mathrm{~g}$ en moyenne, soit des besoins en énergie de 4,1 ; 2,8 et 2,7 UFL/jour/génisse pour les essais I, II et III respectivement.

\section{RÉSULTATS}

\section{Composition chimique du fourrage proposé, quantités ingérées et excrétées, digestibilités}

Le vieillissement du fourrage proposé a surtout affecté sa teneur en MAT qui a diminué en moyenne de 0,13 point par jour de repousse (tableau I). Les taurillons ont ingéré en moyenne $4,88 \mathrm{~kg}$ de MO par jour (tableau II). Les quantités ingérées, exprimées en $\mathrm{kg} \mathrm{MO/jour,} \mathrm{n'ont} \mathrm{pas} \mathrm{diminué} \mathrm{de}$ façon significative avec l'âge de repousse. Rapportées au poids métabolique, elles ont diminué de $0,18 \mathrm{~g}$ par jour de repousse $(p<0,001)$. Les quantités ingérées ont varié significativement d'un taurillon à l'autre $(p<0,001$, tableau II) de 3,9 à $5,3 \mathrm{~kg}$ de MO.

Les quantités journalières de fèces émises ont été de $1,76 \mathrm{~kg}$ de $\mathrm{MO}$ en moyenne par taurillon (tableau II). Elles ont augmenté avec l'âge des repousses $(p<$ $0,001)$ et ont varié entre taurillons ( $p<$ 0,0001 ) de 1,42 à $1,95 \mathrm{~kg}$ MO. La digestibilité de la $\mathrm{MO}$ a été en moyenne de 0,637 (tableau II). Elle a diminué de 0,0027 unité par jour de repousse. La DIV du proposé (tableau I) a été inférieure de 0,08 unité par rapport à la dMO $(p<0,05)$ et a diminué de 0,0017 unité de digestibilité par jour de repousse. 
Tableau I. Composition chimique moyenne et digestibilité in vitro du Dichanthium distribué aux taurillons.

$\begin{array}{lcccc}\text { Variables } & \text { Moyennes } & \begin{array}{c}C V \\ (\%)\end{array} & \begin{array}{r}\text { Effet } \\ \text { agge de } \\ \text { repousse }\end{array} & \begin{array}{r}\text { Pente a } \\ \text { âge de } \\ \text { repousse }\end{array} \\ \text { MSp }(\%) & 20,62 & 7,23 & \text { NS } & 0,009 \\ \text { MOp }(\% \mathrm{MS}) & 88,21 & 0,96 & \star \star & 0,038 \\ \text { MATp } \% \mathrm{MO}) & 10,97 & 19,97 & * \star & -0,127 \\ \text { NDF } p(\% \mathrm{MO}) & 78,76 & 1,99 & \star \star & 0,074 \\ \text { ADFp }(\% \mathrm{MO}) & 42,21 & 3,84 & \star \star & 0,086 \\ \text { DIVp } & 0,55 & 6,66 & & -0,00174\end{array}$

MS $p$, MOp, MAT $p, N D F p$, ADF $p$ : teneurs respectives en matière sèche, matière organique, matières azotées totales, parois totales et lignocellulose du fourrage proposé ; DIV $p$ : digestibilité in vitro du fourrage proposé ; CV : coefficient de variation; a évolution de la variable par jour de repousse; ${ }^{\star \star}: p<0,001$; NS : non significatif.

\section{Equations de prédiction de la dMO}

Les équations significatives de prédiction de la dMO obtenues sont rapportées dans le tableau III. Parmi les covariables testées, MAT $f$ a été le prédicteur le plus précis de la dMO. La précision de la prédiction a été semblable avec l'équation linéaire (équa-

Tableau II. Quantités ingérées, excrétées, composition chimique des fèces et digestibilité de la MO mesurées sur les taurillons à l'auge.

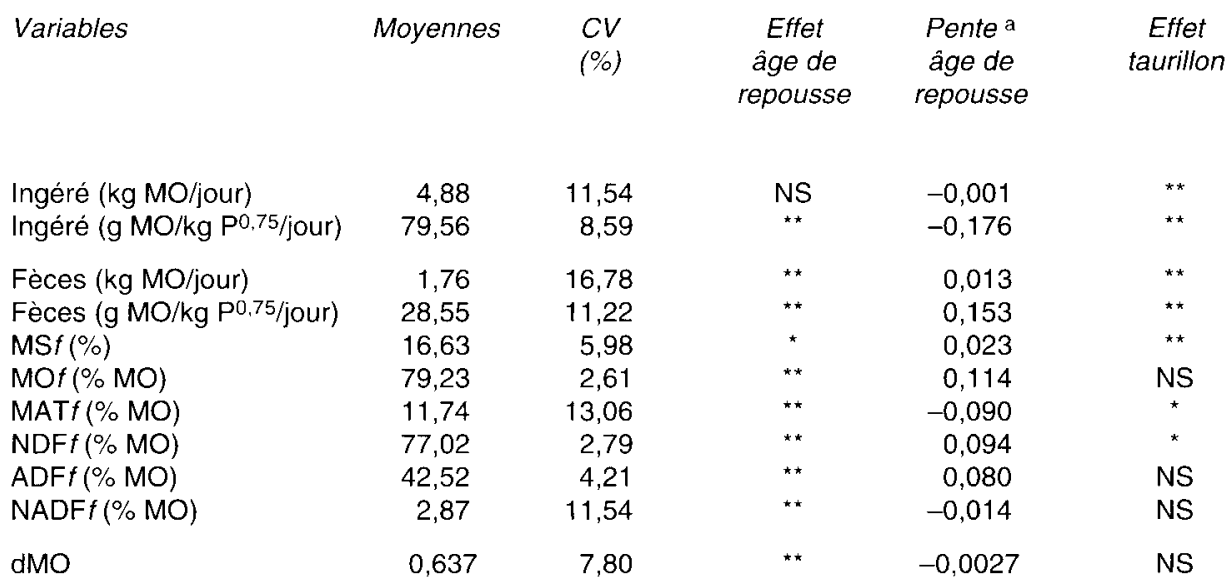

MSf, MOf, MATf, NDF $f$, ADF $f$, NADF $f$ : teneurs respectives en matière sèche, matière organique, matières azotées totales, parois totales, lignocellulose et azote contenu dans l'ADF des fèces ; dMO : digestibilité de la matière organique ; CV : coefficient de variation; a évolution de la variable par jour de repousse ; ${ }^{\star} p<0,01$, ${ }^{\star \star} p<0,001 ; N S$ : non significatif. 
Tableau III. Équations de prédiction de la digestibilité de la matière organique (dMO) à partir de la teneur en constituants fécaux ( $\% \mathrm{MO})$.

\begin{tabular}{|c|c|c|c|c|c|c|c|}
\hline \multirow[t]{2}{*}{ Modèles } & \multirow[t]{2}{*}{$\begin{array}{c}E T R \\
\left(\times 10^{2}\right)\end{array}$} & \multirow[t]{2}{*}{$\begin{array}{l}C V \\
(\%)\end{array}$} & \multirow[t]{2}{*}{$\mathrm{R}^{2}$} & \multirow[t]{2}{*}{$d d l$} & \multicolumn{3}{|c|}{$\begin{array}{l}\text { Effet taurillon } \\
\quad(P>T)\end{array}$} \\
\hline & & & & & Int & $\operatorname{Var} 1$ & Var 2 \\
\hline - & & & & & & & .. \\
\hline 1A) $0,292+0,029 \mathrm{MATf}$ & 2,43 & 3,8 & 0,83 & 37 & 0,71 & 0,79 & \\
\hline 1B) $0,282+0,030 \mathrm{MATf}$ & 1,49 & 2,3 & 0,92 & 7 & & & \\
\hline 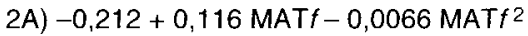 & 2,53 & 3,9 & 0,84 & 37 & 0,98 & 0,99 & 0,99 \\
\hline 2B) $-0,459+0,158 \mathrm{MAT} f-0,0054 \mathrm{MAT} f^{2}$ & 1,19 & 1,9 & 0,94 & 7 & & & \\
\hline 3А) $0,983-4,002 / \mathrm{MATf}$ & 2,30 & 3,6 & 0,84 & 37 & 0,54 & 0,43 & \\
\hline ЗВ) $0,996-4,139 / \mathrm{MAT} f$ & 1,20 & 1,9 & 0,94 & 7 & & & \\
\hline 4A) $2,540-0,024 \mathrm{NDF} f$ & 2,68 & 4,2 & 0,79 & 37 & 0,25 & 0,27 & \\
\hline 4B) $2,748-0,027 \mathrm{NDF} f$ & 1,36 & 2,1 & 0,92 & 7 & & & \\
\hline 5A) $1,730-0,025$ ADF $f$ & 3,43 & 5,4 & 0,65 & 37 & 0,53 & 0,51 & \\
\hline 5B) $1,826-0,027 \mathrm{ADF} f$ & 2,67 & 4,2 & 0,71 & 7 & & & \\
\hline
\end{tabular}

MATf, NDFf, ADFf: teneurs (\% MO) respectives en matières azotées totales, parois totales et lignocellulose dans les fèces. Équations $\mathrm{A}$ : calculées à partir des données individuelles. Équations $\mathrm{B}$ : calculées à partir des valeurs moyennes calculées par semaine pour 5 taurillons. CV : coefficient de variation. Int : seuil de signification de l'effet individu sur l'ordonnée à l'origine. Var 1 et Var 2 : seuils de signification de l'effet taurillon sur la pente du 1er paramètre et du $2^{e}$ paramètre de la régression.

tion $1 \mathrm{~A}$ ), quadratique (équation $2 \mathrm{~A}$ ) et hyperbolique (équation $3 A$ ). Les teneurs en constituants pariétaux des fèces ont été des covariables moins précises que MAT $f$. NDF $f$ a néanmoins permis une précision plus grande qu'ADFf (équations 4A et 5A). L'utilisation simultanée des constituants pariétaux ou de NADFf en plus de MATfn'a pas permis d'améliorer la précision par rapport à MATf seul.

L'effet taurillon et les interactions taurillons $x$ covariables n'ont pas été significatifs $(p>0,05)$ sur les paramètres d'aucune équation de prédiction de la dMO (tableau III). Aussi les paramètres des régressions calculées à partir des données moyennes pour les cinq taurillons (équations $B$, tableau III) sont très semblables à ceux des modèles intégrant toutes les données individuelles (équations $\mathrm{A}$, tableau III).

La corrélation entre dMO et DIV $p$ a été significative et la pente de l'équation n'est pas significativement différente de 1 ( $\mathrm{dMO}=$ $0,022+1,099 \times$ DIVp $; n=8 ; R^{2}=0,72$; $\mathrm{ETR}=0,026)$. L'utilisation de MAT $p$ améliore sensiblement la précision ( $\mathrm{dMO}=0,250$ $+0,420$ DIV $p+0,014$ MAT $p ; R^{2}=0,85$; $\mathrm{ETR}=0,019$ ). Celle-ci reste cependant moins bonne que celle obtenue avec MAT $f$ dans le cas des équations ayant le même nombre de ddl (équations $1 \mathrm{~B}, 2 \mathrm{~B}$ et $3 \mathrm{~B}$ ). L'utilisation d'équations mixtes utilisant simultanément MAT $f$ et DIV $p(E T R=0,013)$ n'améliore pas la précision par rapport à MAT $f$ seul $(E T R=0,012$ pour les équations 2B ou $3 \mathrm{~B}$ du tableau III). 


\section{Équations de prédiction des quantités de MO ingérées (MOI)}

La quantité de MAT excrétée dans les fèces a permis de prédire avec une bonne précision les quantités individuelles de MOI $(n=38 ; E T R=240 \mathrm{~g}$, équation $6 \mathrm{~A}$, tableau IV). La précision a été la même avec une équation du type $a-b / q M A T f$ (équation 7A). Cependant, contrairement aux équations de prédiction de la dMO, l'effet taurillon a été significatif $(p<0,05)$ sur les paramètres de ces deux équations. Les équations calculées à partir des données moyennes des cinq taurillons (équations $B$, tableau IV) ont ainsi conduit à des paramètres sensiblement différents de ceux des équations établies à partir de l'ensemble des données (équations A, tableau IV).

\section{Validation des équations de prédiction de dMO et de MOI au pâturage}

L'analyse a été effectuée à partir des équations intégrant MATf ou qMAT $f$ (équations
1A, 2A, 3A, 6A et 7A). Les teneurs en MAT $f$ de l'essai III (12,7\% MO en moyenne) ont été comprises dans la gamme de variation ayant servi à établir les équations, mais MATf a été plus élevée pour les essais l et II (respectivement 16,5 et 15,5\% MO).

Ces équations ont conduit à des estimations de la digestibilité et des quantités de MO et d'énergie ingérées au pâturage significativement différentes pour un même essai (tableau V). Néanmoins, quelle que soit l'équation de prédiction utilisée, les traitements expérimentaux ont toujours été classés dans le même ordre (les interactions entre les méthodes de calcul et les traitements ont été non significatives dans tous les essais). Par rapport aux besoins énergétiques calculés des animaux, l'équation $1 \mathrm{~A}$ a fortement surestimé les apports dans les essais I et II (fig 1). De même, les équations $6 \mathrm{~A}$ et $7 \mathrm{~A}$ ont surestimé les apports énergétiques dans les essais II et III. L'équation 2A a sous-estimé les apports dans l'essai I. L'équation $3 \mathrm{~A}$ a conduit à des estimations cohérentes bien que toujours très légèrement supérieures aux besoins des animaux.

Tableau IV. Équations de prédiction des quantités de matière organique ingérées ( $\mathrm{MOI}, \mathrm{kg} \mathrm{MO} / \mathrm{jour}$ ) à partir de la quantité de matières azotées totales excrétée dans les fèces (qMATf, kg MO).

\begin{tabular}{|c|c|c|c|c|c|c|}
\hline \multirow[t]{2}{*}{ Modèles } & \multirow[t]{2}{*}{ ETR } & \multirow[t]{2}{*}{$\begin{array}{l}C V \\
(\%)\end{array}$} & \multirow[t]{2}{*}{$\mathrm{R}^{2}$} & \multirow[t]{2}{*}{$d d l$} & \multicolumn{2}{|c|}{$\begin{array}{c}\text { Effet taurillon } \\
\quad(P>T)\end{array}$} \\
\hline & & & & & Int & Var \\
\hline- & & & & & & \\
\hline 6A) $2,327+12,590$ qMATf & 0,240 & 4,9 & 0,86 & 37 & 0,03 & 0,03 \\
\hline 6B) $0,667+20,63$ qMATf & 0,148 & 3,0 & NS & 7 & & \\
\hline 7A) $7,410-0,519 /$ qMATf & 0,239 & 4,8 & 0,86 & 37 & 0,02 & 0,02 \\
\hline 7B) $7,603-0,542 / \mathrm{qMAT} f$ & 0,170 & 3,4 & NS & 7 & & \\
\hline
\end{tabular}

qMATf : quantité ( $\mathrm{kg} \mathrm{MO}$ ) de matières azotées totales dans les fèces. Équations $\mathrm{A}$ : calculées à partir des données individuelles; Équations B : calculées à partir des valeurs moyennes calculées par semaine pour cinq taurillons; $\mathrm{CV}$ : coefficient de variation; Int : seuil de signification de l'effet individu sur l'ordonnée à l'origine; Var : seuil de signification de l'effet taurillon sur la pente du 1 er paramètre de la régression; NS : non significatif. 
Tableau V. Digestibilités ( $\mathrm{dMO}$ ) et quantités ingérées ( $\mathrm{MOI}, \mathrm{kg} \mathrm{MO} / \mathrm{jour}$ ) estimées par les équations $1 \mathrm{~A}, 2 \mathrm{~A}, 3 \mathrm{~A}, 6 \mathrm{~A}$ et $7 \mathrm{~A}$ des tableaux III et IV pour des génisses créoles au pâturage.

\begin{tabular}{|c|c|c|c|c|c|c|}
\hline Essail & & & ssai II & & & sai III \\
\hline $\begin{array}{l}\text { ETR } \\
\left(\times 10^{2}\right)\end{array}$ & $\star \star \star *$ & Moy & $\begin{array}{c}\text { ETR } \\
\left(\times 10^{2}\right)\end{array}$ & $\star \star *$ & Moy & $\begin{array}{l}E T R \\
\left(x 10^{2}\right)\end{array}$ \\
\hline
\end{tabular}

$\begin{array}{cccccccccc}\mathrm{dMO}_{1 \mathrm{~A}} & 0,77 & 1,3 & \mathrm{a} & 0,74 & 1,0 & \mathrm{ab} & 0,66 & 1,2 & \mathrm{~b} \\ \mathrm{dMO}_{2 \mathrm{~A}} & 0,70 & 0,6 & \mathrm{c} & 0,69 & 0,4 & \mathrm{c} & 0,67 & 0,7 & \mathrm{~b} \\ \mathrm{dMO}_{3 \mathrm{~A}} & 0,74 & 0,7 & \mathrm{~b} & 0,72 & 0,6 & \mathrm{~b} & 0,66 & 0,9 & \mathrm{~b} \\ \mathrm{dMO}_{6 \mathrm{~A}} & 0,74 & 0,7 & \mathrm{~b} & 0,76 & 1,2 & \mathrm{a} & 0,71 & 1,0 & \mathrm{a} \\ \mathrm{dMO}_{7 \mathrm{~A}} & 0,73 & 0,7 & \mathrm{~b} & 0,75 & 1,0 & \mathrm{ab} & 0,69 & 0,7 & \mathrm{a} \\ \mathrm{MOI}_{1 \mathrm{~A}} & 6,3 & 0,6 & \mathrm{a} & 4,3 & 0,4 & \mathrm{ab} & 3,7 & 0,4 & \mathrm{c} \\ \mathrm{MOI}_{2 \mathrm{~A}} & 4,6 & 0,2 & \mathrm{c} & 3,5 & 0,3 & \mathrm{c} & 3,8 & 0,3 & \mathrm{bc} \\ \mathrm{MOI}_{3 \mathrm{~A}} & 5,3 & 0,3 & \mathrm{~b} & 3,9 & 0,3 & \mathrm{bc} & 3,8 & 0,4 & \mathrm{c} \\ \mathrm{MOI}_{6 \mathrm{~A}} & 5,2 & 0,1 & \mathrm{~b} & 4,4 & 0,9 & \mathrm{a} & 4,3 & 0,2 & \mathrm{a} \\ \mathrm{MOI}_{7 \mathrm{~A}} & 5,1 & 0,1 & \mathrm{~b} & 4,2 & 0,3 & \mathrm{ab} & 4,1 & 0,3 & \mathrm{ab}\end{array}$

** Effet du modèle utilisé sur l'estimation de la dMO, des MOl et des UFV ingérès. Les valeurs d'une même colonne suivies de la même lettre ne sont pas significativement différentes à $p<0,05$, par le test de Scheffe. Les différentes équations utilisées pour estimer $\mathrm{dMO}$ et MOI sont : $\mathrm{dMO}_{1 \mathrm{~A}}=0,292+0,029 \mathrm{MAT} ; \mathrm{dMO}_{2 \mathrm{~A}}=-0,212+0,116 \mathrm{MAT} f$ $-0,0036 \mathrm{MAT}^{2} ; \mathrm{dMO}_{3 \mathrm{~A}}=0,983-4,002 / \mathrm{MAT} ; \mathrm{dMO}_{6 \mathrm{AA}}=\left(\mathrm{MOI} \mathrm{I}_{6 \mathrm{~A}}-\mathrm{MOF}\right) / \mathrm{MOI}_{6 \mathrm{AA}} ; \mathrm{dMO}_{7 \mathrm{~A}}=\left(\mathrm{MOI}_{7 \mathrm{~A}}-\mathrm{MOF}\right) / \mathrm{MOI}_{7 \mathrm{~A}} ;$ $\mathrm{MOI}_{1 \mathrm{~A}}=\mathrm{MOF} /\left(1-\mathrm{dMO}_{1 \mathrm{~A}}\right) ; \mathrm{MO}_{2 \mathrm{~A}}=\mathrm{MOF} /\left(1-\mathrm{dMO}_{2 \mathrm{~A}}\right) ; \mathrm{MOI}_{3 \mathrm{~A}}=\mathrm{MOF} /\left(1-\mathrm{dMO}_{3 \mathrm{~A}}\right) ; \mathrm{MOI}_{6 \mathrm{~A}}=2,327+12,59 \mathrm{qMAT} f$ $\mathrm{MOI}_{7 \mathrm{~A}}=7,410-0,519 / \mathrm{qMAT} f$.

\section{DISCUSSION}

\section{Valeur alimentaire du Dichanthium sp}

La composition chimique du Dichanthium $\mathrm{sp}$ est comparable à celle d'autres fourrages tropicaux (Vera et al, 1973; Mackenzie et al, 1982 ; Tudor et Minson, 1982 ; Minson, 1984 ; Aumont et al, 1995). La teneur en matières azotées ne dépasse pas $9 \%$. Les teneurs en parois sont élevées et la digestibilité de la matière organique est, au mieux, égale à 0,68 pour des repousses de 20 jours. L'ingestibilité de Dichanthium sp diminue peu avec l'âge des repousses $(-0,18$ $\mathrm{g} / \mathrm{kg} \mathrm{P0,75/jour,} \mathrm{soit} 0,2 \%$ ), comme cela a déjà été montré pour d'autres espèces tropicales (Milford et Minson, 1965 ; Jeffery, 1971 ; Minson, 1972). Des diminutions plus rapides ont été mesurées en zone tempérée

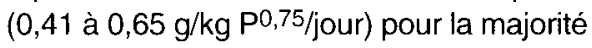
des prairies naturelles à base de graminées (Demarquilly et al, 1981a).

La digestibilité du Dichanthium sp diminue de 0,0027 unité/jour de repousse, comme celle d'autres fourrages tropicaux (Milford et Minson, 1965 ; Olubajo et Oyenuga, 1970 ; Vera et al, 1973 ; Chenost, 1975). Cette diminution est plus faible que celle observée pour des graminées au premier cycle ou pour des repousses à tige en zone tempérée, pour lesquelles la digestibilité diminue de 0,0035 à 0,0047 unité/jour de repousse (Demarquilly et Jarrige, 1981). Selon Chenost (1975), cette évolution modérée de la digestibilité avec l'âge s'explique par le fait que la teneur en parois des fourrages tropicaux est déjà élevée chez les repousses jeunes et que ces parois se ligni- 


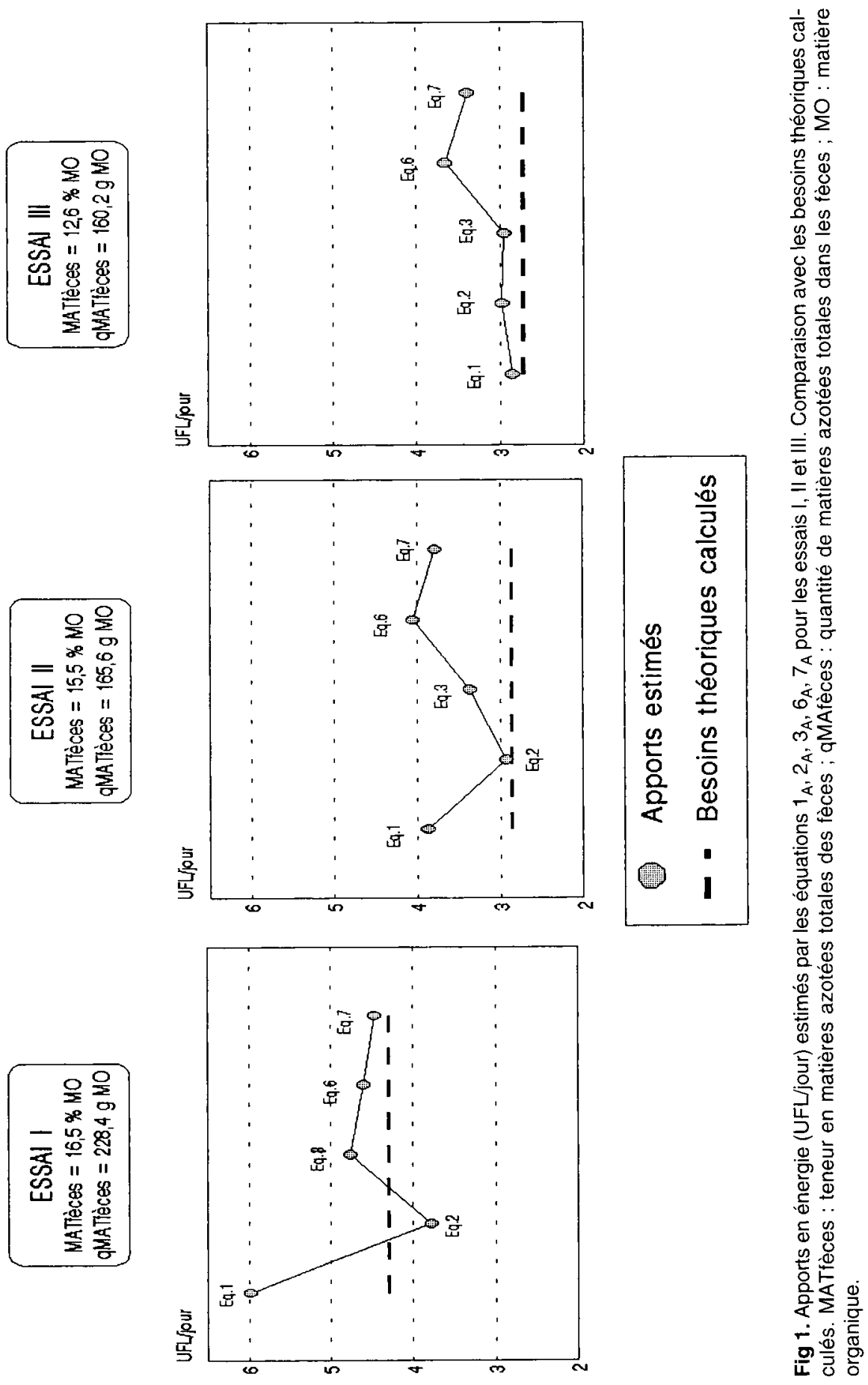


fient très tôt. Par la suite, la teneur en paroi et leur lignification n'augmentent que lentement avec le vieillissement du fourrage. En revanche, la teneur en MAT diminue nettement avec l'âge de repousse (Chenost, 1975 ; Aumont et al, 1995), parallèlement à la diminution du rapport feuille/tige.

\section{Équations de prédiction de la digestibilité}

La précision de la prédiction de $\mathrm{dMO}$ à partir de la digestibilité in vitro du fourrage proposé a été supérieure à celle obtenue par d'autres auteurs (Mc Leod et Minson, 1969, 1974) mais est restée inférieure à celle obtenue par les index fécaux et MATf en particulier (équations B du tableau III). Ceci peut s'expliquer en partie par un tri effectué à l'auge par les animaux qui étaient alimentés à volonté bien que le fourrage ait été préalablement haché. II ne semble donc pas souhaitable de proposer cette méthode d'estimation de la digestibilité dans les essais conduits au pâturage où l'application d'une telle équation nécessite en plus le prélèvement d'échantillons représentatifs du fourrage réellement ingéré par l'animal (Holechek et al, 1982), ce qui la rend encore plus difficile d'emploi. Ce problème d'échantillonnage de l'herbe n'existe plus dans le cas des équations basées sur les indicateurs fécaux.

Les équations de prédiction de la dMO à partir de la teneur en MATf ont des précisions satisfaisantes quand elles sont comparées aux équations du même type établies en zone tropicale avec des fourrages verts. Olubajo et Oyenuga (1970) ont obtenu des ETR faibles $(0,005$ à 0,0219 unité) pour des équations établies à différentes périodes de l'année. Jeffery (1971) et Vera (1973) ont établi des régressions simples ou quadratiques avec des ETR supérieurs à ceux de nos équations $(0,038$ à 0,046 unité). Comparativement aux équa- tions établies en zone tempérée, la précision de nos équations est également satisfaisante. Greenhaigh et Corbett (1960a) et Thomas et Campling (1976) ont établi des régressions linéaires avec des observations moyennes et ont obtenu des ETR de 0,015 unité.

MATfest l'indicateur fécal le plus précis. La prédiction de la dMO à partir des constituants pariétaux des fèces est moins précise qu'avec MAT $f$ comme l'ont aussi montré Vera (1973) et Holloway et al (1981). En outre le dosage de ces constituants est plus long et moins précis que celui de MATf. De plus, l'addition d'autres consituants fécaux ou de la DIV $p$ à MAT $f$ n'a pas permis d'améliorer la précision de la prédiction comme cela avait déjà été observé pour des équations de prédiction établies à partir d'une population de fourrages plurispécifiques, associant notamment des graminées et des légumineuses (Holloway et al, 1981 ; Guerin et al, 1988 ; Comeron, 1991).

En utilisant MATf comme seul indicateur fécal, Greenhalgh et Corbett (1960b) et Bartiaux-Thill et Oger (1986) ont obtenu une précision meilleure avec des modèles quadratiques qu'avec des modèles linéaires. Cela n'a pas été le cas dans notre étude, où seule l'équation de type hyperbolique a conduit à une précision légérement plus élevée que les deux autres équations. L'équation hyperbolique a par ailleurs permis d'estimer des apports énergétiques plus cohérents avec les besoins des animaux lorsqu'elle a été utilisée au pâturage avec une large gamme de qualité de fourrage et de situations nutritionnelles des génisses. Elle apparaît donc plus pertinente. En fait, l'équation de type hyperbolique repose sur le fait que la quantité d'azote excrétée dans les fèces est relativement constante pour une quantité fixée de MOI. En effet, à partir d'une population de 1208 graminées de prairies naturelles utilisées en vert, Demarquilly et al (1981b) ont montré que la teneur en matières azotées digestibles de l'ingéré 
(MADi, \% MO) est liée de manière étroite et linéaire à la teneur en MATi (matières azotées totales de l'ingéré) (\% MO) :

$\mathrm{MADi}=0,931 \mathrm{MAT} i-3,98(r=0,994)$ (éq 1) équation qui montre que la digestibilité vraie des protéines des fourrages verts est très élevée $(0,93)$ et que les matières azotées fécales sont essentiellement d'origine microbienne et endogène. Ainsi, les quantités de matières azotées excrétées (qMAT $f=$ (MAT $i$ - MADi) x MOI)) sont peu affectées par la teneur en MAT du fourrage et elles varient essentiellement avec MOI

qMAT $f=(0,069 \mathrm{MAT} i+3,98) \times$ MOI (éq 2$)$.
En posant qMAT $f=\mathrm{MOI} \times(1-\mathrm{dMO}) \times$ MAT $f$ dans l'équation 2, il vient :

$\mathrm{dMO}=1-0,069$ MAT $/$ MAT $f-3,98 /$ MATf qui peut se simplifier sous la forme

$\mathrm{dMO}=1-b /$ MAT $f$

ou $b$ représente essentiellement la quantité de matières azotées d'origine non alimentaire excrétée par unité de MOI. Cette équation de type hyperbolique repose donc sur des bases physiologiques. C'est pourquoi, elle est apparue beaucoup plus robuste que les modèles linéaires ou quadratiques qui ne sont plus valables lorsque ces équations sont utilisées en dehors de leur domaine de définition dans les essais pâturage. De

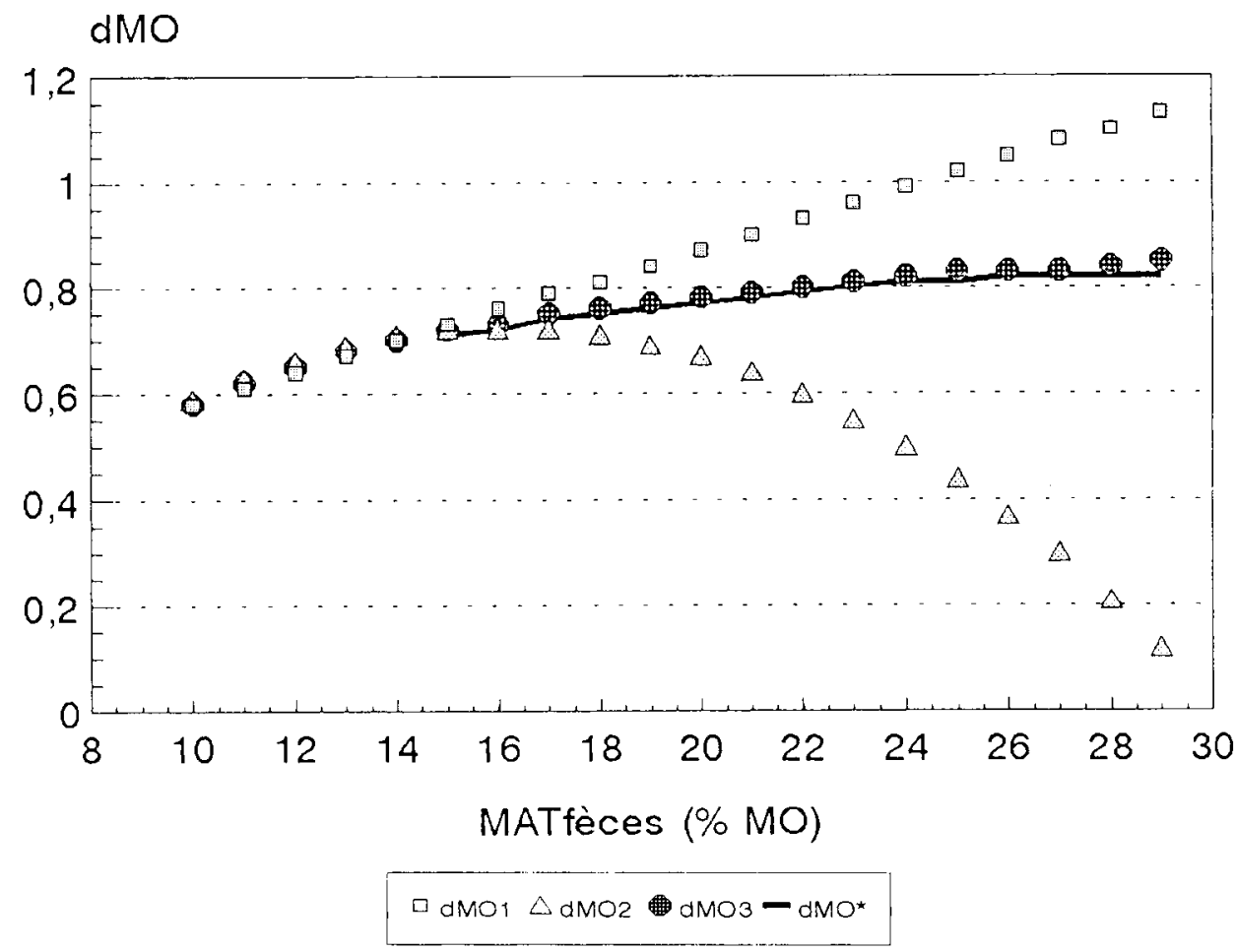

Fig 2. Évolution de la digestibilité de la matière organique (dMO) estimée par les équations $1_{A}, 2_{A}$ ou $3_{\mathrm{A}}$ (dMO1, dMO2 et $\mathrm{dMO} 3$ respectivement) et par une équation établie en zone tempérée (dMO ${ }^{\star}$ ) à partir de 71 mesures provenant de trois équipes de recherches en fonction de la teneur en matières azotées totales des fèces (MATf) (Peyraud, non publié). ( $\mathrm{dMO}^{*}=0,33+0,038 \mathrm{MAT} f-0,0006 \mathrm{MATf}$ $(E T A=0,015 ; n=71)$. 
même, le modèle linéaire conduirait à surestimer fortement les digestibilité des fourrages de bonne qualité des zones tempérées qui sont caractérisés par des teneurs en MATf élevées ( $20 \%$ ) tandis que le modèle curvilinéaire conduirait à des digestibilités qui diminueraient pour MATf supérieure à $17 \% \mathrm{MO}$. En revanche, l'équation hyperbolique permet de prédire sans biais excessif la digestibilité des graminées des zones tempérées beaucoup plus digestibles (fig 2). Une telle équation avait déjà été proposée par Lancaster (1949b) mais n'avait jamais été reprise depuis lors.

\section{Équations de prédiction des quantités ingérées}

La quantité de MAT excrétée est un prédicteur précis de MOI (240 g MO avec $n=37$, tableau IV). La précision de la régression simple entre MOl et qMATf est supérieure à celle des régressions établies pour un fourrage donné pour des taurillons (Leite et Stuth, 1990) ou des moutons (Jeffery, 1971 ; Aumont et al, soumis pour publication). Cependant, contrairement aux équations de prédiction de la dMO, les paramètres de ces équations varient entre taurillons. Cela restreint l'utilisation de tels modèles pour estimer des variations individuelles de l'ingestion au pâturage.

\section{CONCLUSION}

Pour estimer la digestibilité et les quantités ingérées de Dichanthium sp par des bovins créoles, la teneur en MAT des fèces est l'indicateur le plus précis. La précision des équations de prédiction mises au point dans nos conditions tropicales est très satisfaisante par rapport à des équations établies en zone tempérée et dans d'autres zones tropicales. L'équation de prédiction de la digestibilité du type hyperbolique est celle qui conduit à des estimations les plus cohérentes au pâturage, y compris lorsqu'elle est utilisée en dehors de la gamme de digestibilité dans laquelle elle a été établie. Cette équation est bien adaptée à l'approfondissement des études des facteurs de variation de l'ingestion au pâturage en conditions tropicale.

\section{REMERCIEMENTS}

Nous remercions toute l'équipe du domaine de Gardel et plus particulièrement $P$ Mulciba et A Nepos pour leur contribution aux mesures à l'auge. Nous remercions également toute l'équipe du laboratoire de Duclos, pour les nombreuses analyses réalisées dans le cadre de ce travail. Enfin nous remercions $\mathrm{H}$ Archimède pour sa grande disponibilité lors des corrections de cet article.

\section{RÉFÉRENCES}

Aumont G, Caudron I, Xande A (1991) Tables des valeurs alimentaires de fourrages tropicaux de la zone Caraibe et de la Réunion (Inra-SRZ, ed), Guadeloupe, $199 \mathrm{p}$

Aumont G, Caudron I, Saminadin G, Xande A (1995) Sources of variation in nutritive values of tropical forages from the Caribbean. Anim Feed Sci Tech $51,1-13$

Bartiaux-Thill N, Oger R (1986) The indirect estimation of the digestibility in cattle of herbage from Belgian permanent pasture. Grass Forage Sci 41, 269272

Berbigier $P$ (1988) Bioclimatologie des ruminants domestiques en zone tropicale. Inra Éd, Paris, $135 \mathrm{p}$

Chenost M (1975) La valeur alimentaire du pangola (Digitatia decumbens stent) et ses facteurs de variation, en zone tropicale humide. Ann Zootech 24, 327-349

Chenost M (1985) Estimation de la digestibilite de l'herbe ingérée au pâturage à partir de l'azote fécal et de quelques autres paramètres fécaux. Ann Zootech 34, 205-228

Comeron EA (1991) Estimation des quantités ingérées des vaches laitières au pâturage : influence des caractéristiques des animaux et influence de la qualitè et de la structure de l'herbe offerte. Thèse de doctorat, Ensa Rennes, France, 156 p 
Cordova FJ, Wallace JD, Pieper RD (1978) Forage intake by grazing livestock: a review. J Range Manage 31, 430-438

Demarquilly C, Andrieu J, Weiss Ph (1981a) L'ingestibilité des fourrages verts et des foins et sa prévision. In: Prévision de la valeur nutritive des aliments des ruminants, Inra Éd, Versailles, 155-167

Demarquilly C, Grenet E, Andrieu J (1981b) Les constituants azotés des fourrages et la prévision de la valeur azotée des fourrages. In : Prévision de la valeur nutritive des aliments des ruminants, Inra Éd, Versailles, 129-154

Demarquilly $C$, Jarrige $R$ (1981) Panorama des méthodes de prévision de la digestibilité et de la valeur énergétique des fourrages. In: Prévision de la valeur nutritive des aliments des ruminants, Inra Ed, Versailles, 41-59

Doves H, Mayes RW (1991) The use of plant Wax alkanes as marker substances in studies of the nutrition of herbivores: a review. Aust $J$ Agric Res 42, 913-952

Greenhalgh JFD, Corbett JL (1960a) The indirect estimation of the digestibility of pasture herbage. I. Nitrogen and chromogen as faecal index substances. J Agric Sci Camb 55, 371-376

Greenhalgh JFD, Corbett JL (1960b) The indirect estimation of the digestibility of pasture herbage. II. Regressions of digestibility on faecal nitrogen concentration ; their determination in continuous digestibility trials and the effect of various factors on their accuracy. J Agric Sci Camb 55, 377-386

Greenhalgh JFD (1982) An introduction to herbage intake measurements. In : Herbage Intake Handbook (JD Leaver, ed), British Grassland Society, 1-10

Guérin H, Richard D, Friot D, Mbaye Nd, Kone AR (1988) Intérêt du dosage de la lignocellulose (ADF) et de différentes fractions azotées pour prévoir la valeur nutritive des fourrages naturels sahéliens. Reprod Nutr Dév 28, suppl 1, 111-112

Holechek JL, Vavra M, Pieper RD (1982) Methods for determining the nutritive quality of range ruminant diets: a review. J Anim Sci 54, 354-376

Holloway JW, Estell RE, Butts WT (1981) Relationship between fecal components and forage consumption and digestibility. J Anim Sci 52, 836-848

Hutton JB, Jury KE (1964) Studies of the nutritive value of New Zealand dairy pastures. III. The comparative value of various feed-faeces relationships in herbage intake studies with dairy cattle. New Zealand $J$ Agric Res 7, 583-595

Jarrige R (1965) La composition des fèces de mouton et ses relations avec la digestibilité des fourrages. Fourrages $22,1-13$

Jeffery $H$ (1971) Assessment of faecal nitrogen as an index for estimating digestibilty and intake of food by sheep on Pennisetum clandestidum based pasture. Aust J Exp Agric Anim Husb 11, 393-396
Judkins MB, Krysl LJ, Barton RK (1989) Estimating diet digestibility: a comparison of 11 techniques across 6 different diets fed to rams. J Anim Sci $68,1405^{-}$ 1415

Lancaster RJ (1949a) Estimation of digestibility of grazed pasture from faeces nitrogen. Nature 163, 330331

Lancaster RJ (1949b) The measurement of feed intake by grazing cattle and sheep. I. A method of calculating the digestibility of pasture based on the nitrogen content of feces derived from the pasture. New Zealand J Sci Tech 31, 31-38

Le Du YLP, Penning PD (1982) Animal based techniques for estimating herbage intake. In : Herbage Intake Handbook (JD Leaver, ed), British Grasslands Society, 37-75

Leite ER, Stuth JW (1990) Value of multiple fecal indices for predicting diet quality and intake of steers. $J$ Range Manage 43, 139-143

Mc Leod MN, Minson DJ (1969) Sources of variation in the in vitro digestibility of tropical grasses. $J \mathrm{Br}$ Grassid Soc 24, 244-249

Mc Leod MN, Minson DJ (1974) Predicting organic matter digestibility from in vivo and in vitro determination of dry matter digestibility. $J \mathrm{Br}$ Grassid Soc 29, $17-21$

Mackenzie J, Mayer R, Bisset WJ (1982) Productivity of five subtropical grasses on a black earth of the eastern darling downs of Queensland. Trop Grass 16. 171-179

Milford R, Minson DJ (1965) Intake of tropical pasture species. 9th Proc Int Grassl Congr, 815-822

Minson DJ (1972) The digestibility and voluntary intake by sheep of six tropical grasses. Aust J Exp Agric Anim Husb 12, 21-26

Minson DJ (1984) Digestibility and voluntary intake by sheep of 5 Digitaria species. Aust J Exp Agric Anim Husb 24, 494-500

Olubajo FO, Oyenuga VA (1970) Digestibility of tropical pasture mixtures using the indicator technique. $J$ Agric Sci Camb 75, 175-181

Raymond WF, Kemp CD, Kemp AW, Harris CE (1954) Studies in the digestibility of herbage. IV. The use of faecal collection and chemical analysis in pasture studies. (b) Faecal index methods. J Br Grassld Soc 9, 69-82

SAS (1988) SAS user's guide. Statistics. SAS Inst Inc, Cary, NC, États-Unis

Scales GH, Streeter CL, Denham AH, Ward GM (1974) A comparison of indirect methods of predicting in vivo digestibility of grazed forage. J Anim Sci 38, 192-198

Streeter CL (1969) A review of techniques used to estimate the in vivo digestibility of grazed forage. $J$ Anim Sci $29.757-768$ 
Thomas S, Campling RC (1976) Relationship between digestibility and faecal nitrogen in sheep and cows offered herbage. J Br Grassid Soc 31, 69-72

Tilley JMA, Terry RA (1963) A two stage technique for in vitro digestion of forage crops. $J$ Br Grassid Soc 18, 104-111

Troccon JL (1987) Recommandations alimentaires pour les veaux et les génisses d'élevage. Bull Tech CRVZ Theix INRA, 70, 167-172

Tudor GD, Minson DJ (1982) The utilization of the dietary energy of pangola and setaria by young growing beef cattle. J Agric Sci Camb 98, 395-404
Van Soest PJ (1963) Use of detergents in the analysis of fibrous feeds. 2. A rapid method for the determination of fibre and lignin. J Assoc Off Agric Chem 46, 829835

Vera RR (1973) The nutritive value of weeping lovegrass during the spring season. 2 . The use of faecal fibre and nitrogen to predict digestibility. J Br Grassld Soc 28, 243-245

Vera RR, Irazoqui $H$, Menvielle EE (1973) The nutritive value of weeping lovegrass during the spring season. 1. Digestibility and yield of digestible nutrients. $\mathrm{J} \mathrm{Br}$ Grassid Soc 28, 149-152 